\title{
The role of explicit memory processes in cross-modal priming: An investigation of stem completion priming in amnesia
}

\author{
MIEKE VERFAELLIE \\ Memory Disorders Research Center, Boston VA Healthcare System \\ and Boston University School of Medicine, Boston, Massachusetts \\ MARGARET M. KEANE \\ Wellesley College, Wellesley, Massachusetts, \\ Memory Disorders Research Center, Boston VA Healthcare System, \\ and Boston University School of Medicine, Boston, Massachusetts \\ and \\ SHAUN P. COOK \\ Memory Disorders Research Center, Boston VA Healthcare System \\ and Boston University School of Medicine, Boston, Massachusetts
}

\begin{abstract}
To clarify the role of explicit memory processes in cross-modal priming, two experiments examined the status of cross-modal stem completion priming in amnesia. Experiment 1 used a standard behavioral paradigm in which stems corresponding to studied and unstudied words were intermixed. Amnesic patients showed intact within- and cross-modal priming, but, in contrast to controls, they recognized very few of their completions as having been on the study list. This finding suggests that memorial awareness is not necessary for cross-modal priming to occur. Experiment 2 used a paradigm modeled after functional imaging studies, in which stems corresponding to studied and unstudied words were blocked. Amnesic patients showed intact within-modal priming, but impaired cross-modal priming. This finding is consistent with the notion that a blocked format induces voluntary retrievalstrategiesin normal participants.
\end{abstract}

Studies of patients with amnesia have provided some of the most compelling evidence for the existence of forms of memory that are functionally and neuroanatomically dissociable from explicit remembering. One of these forms of memory is priming, the facilitation or bias in identifying or producing an item as a consequence of its prior encounter (Tulving \& Schacter, 1990). Patients with amnesia show intact priming in a variety of paradigms, despite the fact that they have marked deficits in explicit memory for the task stimuli (Moscovitch, Vriezen, \& Gottstein, 1993; Schacter, Chiu, \& Ochsner, 1993).

One form of priming that has been studied extensively is perceptual priming. Perceptual priming tasks require participants to identify or complete perceptually degraded stimuli, such as briefly flashed pictures or word stems. Priming in these tasks varies with the degree of perceptual match between the study and test items. Priming is significantly greater when stimuli are presented in the same sensory modality at study and test (e.g., visual-visual) than

This research was supported by NINDS Program Project Grant NS26985 and NIMH Grant MH57681 to Boston University and by the Medical Research Service of the VA Healthcare System. Correspondence should be addressed to M. Verfaellie, Memory Disorders Research Center (151A), Boston VA Healthcare System, 150 S. Huntington Avenue, Boston, MA 02130 (e-mail: verf@bu.edu). when the sensory modality is changed from study to test (e.g., auditory-visual). Such findings have led to the notion that priming depends on modality-specific perceptual processes (Roediger, Weldon, \& Challis, 1989; Schacter, 1994).

Although changes in modality between study and test greatly reduce priming in perceptual tasks, they do not always eliminate it. Significant priming often persists in a cross-modal condition (Toth \& Reingold, 1996). The nature of this cross-modal priming component is not well understood. One class of explanations has focused on the facilitation of nonperceptual aspects of processing, such as those involved in the activation of semantic codes (Bassili, Smith, \& Macleod, 1989) or phonological codes (Curran, Schacter, \& Galluccio, 1999; Kirsner, Dunn, \& Standen, 1989). Another class of explanationshas focused on the role of explicit memory processes (Jacoby, Toth, \& Yonelinas, 1993; Jacoby, Toth, Yonelinas, \& Debner, 1994; RichardsonKlavehn \& Gardiner, 1996). The goal of the present study was to reconsider the contribution of explicit memory to cross-modal priming in the stem completion task by examining the performance of amnesic patients.

\section{EXPERIMENT 1}

Using the process dissociation procedure, Jacoby et al. (1993) have demonstrated that auditory presentation of 
stimuli results in little or no automatic influence of memory in a visual stem completion task. They argued, therefore, that cross-modal priming reflects contamination by intentional (i.e., explicit) retrieval. One finding that casts doubt on this interpretation, however, is the fact that a levelsof-processing manipulation affects explicit cross-modal stem-cued recall, but not implicit cross-modal stem completion (Craik, Moscovitch, \& McDowd, 1994). According to the retrieval intentionality criterion (Schacter, Bowers, \& Booker, 1989), this pattern of results suggests that the cross-modal stem completion test is free of explicit contamination.

Richardson-Klavehn and Gardiner (1996) have reconciled these contradictory findings by reinterpreting the role of explicit memory in cross-modal priming. They suggested that cross-modal priming does not depend on voluntary retrieval but, rather, is accompanied by involuntary conscious memory. The process dissociation procedure does not distinguish retrieval volition from memorial awareness. Therefore, what was thought to reflect voluntary retrieval in the process dissociation framework might instead reflect involuntary retrieval accompanied by awareness that generated items were on the study list. Support for this notion comes from a study in which participants first completed stems with the first word coming to mind and then indicated whether they were aware that their completion came from the study list (RichardsonKlavehn \& Gardiner, 1996). As in Craik et al. (1994), cross-modal priming was not affected by a levels-ofprocessing manipulation, suggesting that retrieval was unintentional. At the same time, cross-modal priming was associated with memorial awareness, as indicated by participants' recognition of completed stems. In fact, there was no cross-modal priming unassociated with memorial awareness.

At present, it remains unclear whether memorial awareness plays a causal role in cross-modal priming or is simply a correlate of such priming (see, e.g., RichardsonKlavehn \& Gardiner, 1996; Richardson-Klavehn, Gardiner, \& Java, 1994). The study of amnesic patients may provide an answer to this question. If amnesic patients show normal cross-modal priming, but impaired memorial awareness, it would suggest that conscious awareness is not necessary for cross-modal priming. Two studies have provided evidence for intact cross-modal priming in amnesia (Carlesimo, 1994; Graf, Shimamura, \& Squire, 1985), thus providing further evidence that cross-modal priming does not depend on voluntary (i.e., explicit) retrieval. However, neither of these studies directly assessed memorial awareness in the stem completion task. Therefore, the role of memorial awareness associated with involuntary retrieval in cross-modal priming remains unknown. To address this issue, we examined cross-modal priming and associated memorial awareness of completions in patients with amnesia. To document the severity of patients' amnesia, we also administered a stem-cued recall task, which differed from the stem completion task only in terms of retrieval instructions.

\section{Method}

Participants. Sixteen amnesic patients with an etiology of Korsakoff's syndrome $(n=7)$, anoxia $(n=6)$, encephalitis $(n=2)$, or bithalamic stroke $(n=1)$ participated. The amnesic group had a mean age of 60 years and a mean education of 14.6 years. Their intellectual functioning was in the average range, as indicated by a mean verbal IQ score of 100 on the Wechsler Adult Intelligence Scale-III (WAIS-III; Wechsler, 1997a). Their memory functioning was severely compromised: On the Wechsler Memory Scale-III (WAIS-III; Wechsler, 1997b), they obtained a mean general memory index of 59, a mean visual delay index of 63, and a mean auditory delay index of 64 . Their attentional abilities, as measured by the WMS-III working memory index, were average $(M=99)$.

The control group consisted of 7 individuals with a history of alcoholism and 9 healthy adults without a history of alcoholism. Three participants were replaced because, at the end of the experiment, they indicated that they did not give the first word that came to mind if they realized that word had appeared on the study list. Control participants were matched to the amnesic patients in terms of age $(M=$ 61 years), education ( $M=14$ years), and verbal IQ $(M=107)$.

Materials and Design. Stimuli were 128 words with a mean length of 6.1 letters and a mean frequency of 82 (Francis \& Kučera, 1982). The three-letter stem for each word was unique and had at least 10 completions $(M=17.2)$. Two test lists were constructed, each consisting of four sets of 16 words matched for length, frequency, and number of possible stem completions. One list was used for the implicit test and the other for the explicit test. The assignment of lists to test conditions was counterbalanced across participants. For each list, test items were assigned to the visual study, auditory study, and unstudied conditions. The unstudied condition was always composed of two item sets to equate the number of studied and unstudied items. The four sets of items were rotated through these conditions. Each study list consisted of two sets of 16 words, one of which appeared in the visual condition and the other in the auditory condition. The order of presentation of visual and auditory conditions was counterbalanced across participants. Two filler items were added to the beginning and end of each study list to prevent primacy and recency effects.

Procedure. The participants were tested in a single session. The implicit task was always administered first and was separated from the explicit task by a 10-min break. During the study phase of each task, the participants were exposed to two sets of words, one presented auditorily and the other presented visually. The participants were asked to rate the pleasantness of each word on a 5-point scale. Stimulus presentation was controlled by a Macintosh PowerBook G3 computer. Visual stimuli were presented for $2 \mathrm{sec}$ in the center of the screen. Auditory stimuli were recordings that had been normalized and smoothed using SoundEdit 16. For stimuli in both modalities, the intertrial interval was $1 \mathrm{sec}$. A fixation point was presented between the two sets of words to indicate a change in presentation modality. There was approximately a 6-min delay between the study phase and the test phase, during which the participants completed stems with names of cities.

During the implicit test phase, the participants were told that threeletter stems would appear on the screen, and they were to complete each stem with the first word that came to mind. The experimenter recorded their responses. Following the completion phase, the participants were given the list of words that they had just generated and were asked to circle any words that they recognized from the study session.

During the explicit test phase, the participants were asked to complete three-letter stems with words from the study list. They were asked not to guess and to leave stems blank if they could not think of words from the study list.

\section{Results and Discussion}

The results of the alcoholic and nonalcoholic subgroups within the amnesic and control groups were com- 
Table 1

Mean Proportion of Stems Completed With Targets in Experiment 1

\begin{tabular}{llcc}
\hline & \multicolumn{3}{c}{ Study Condition } \\
\cline { 2 - 4 } Group & Visual & Auditory & Unstudied \\
\hline Amnesics & $.36(.06)$ & $.19(.05)$ & $.08(.02)$ \\
Controls & $.38(.14)$ & $.21(.10)$ & $.09(.01)$ \\
\hline
\end{tabular}

Note-The proportion of stems completed with targets and attributed to the study list is given in parentheses.

bined because there were no significant differences as a function of alcohol history.

Implicit stem completion. Table 1 presents the mean proportion of stems completed with targets (with the proportion of stems completed to targets and attributed to the study list between parentheses). Priming was significant in both the visual modality and the auditory modality for both groups (all $t \mathrm{~s}>2.9, p \mathrm{~s}<.01$ ). As expected, priming was greater in the within-modal condition than in the crossmodal condition, and both within-modal priming and cross-modal priming were of similar magnitude in the two groups. An analysis of variance (ANOVA) on priming scores (proportion of studied - unstudied completions) revealed a significant effect of modality $[F(1,30)=30.2$, $p<.01]$, but no effect of group and no group $\times$ modality interaction (both $F_{\mathrm{s}}<1$ ).

Table 1 also lists the proportion of stems that were completed to targets and subsequently recognized as coming from the study list. Recognition of target completions did not differ as a function of modality but was lower for amnesics than for controls. An ANOVA on the proportion of correctly recognized target completions (studied - unstudied target completions that were endorsed) revealed a main effect of group $[F(1,30)=5.3$, $p<.05]$. Neither the effect of modality $[F(1,30)=1.5$, $p>.20]$ nor the group $\times$ modality interaction $[F(1,30)=$ $1.0, p>.30$ ] was significant.

In both groups, there were very few unstudied items that were completed to targets and subsequently (incorrectly) endorsed as having been on the study list. This might lead one to conclude that the false alarm rate in recognition was similar in the amnesic and control groups. However, the false alarm rate was clearly higher in amnesic patients than in controls, when considering the proportion of nontarget completions subsequently (incorrectly) attributed to the study list ( $M=.17$ vs. .03 ). This liberal bias in the amnesic group led to an overestimate of the magnitude of memorial awareness associated with target completions in amnesia.

To compare more accurately memorial awareness across the two groups, we analyzed the recognition of target completions for a subset of patients $(n=11)$ who endorsed nontarget completions as having been on the study list at a rate comparable to controls (patients, $M=.05$; controls, $M=.03 ; t<1)$. This subgroup of patients showed similar target completion rates to the amnesic group as a whole ( within-modal $=.41$, cross-modal $=.20$, unstudied $=.08$ ), but had very few target completions that were associated with memorial awareness (within-modal $=.03$, crossmodal $=.03$, unstudied $=.01$ ).

By subtracting the proportion of target completions associated with memorial awareness from the total proportion of target completions, we obtained a measure of completions not associated with awareness. Table 2 presents these proportions for the controls and the subgroup of amnesics who had a comparable response bias. There was significant within-modal priming not associated with awareness in both the amnesic group and the control group $(t \mathrm{~s}>4.6, p<.01)$. Cross-modal priming unassociated with awareness was not reliable in the control participants, $[t(15)<1]$ but was significant in the amnesic group ${ }^{1}[t(10)=4.0, p<.01]$. As expected, priming unassociated with awareness was greater in the amnesic group than in the control group. Also, for both groups, "unaware" priming was greater in the within-modal condition than in the cross-modal condition. An ANOVA revealed significant effects of group $[F(1,25)=15.4, p<$ $.01]$ and modality $[F(1,15)=36.7, p<.01]$. The group $\times$ modality interaction was not significant $[F(1,25)=2.2$, $p>.14]$.

Explicit stem-cued recall. As can be seen in Table 3, the amnesic patients recalled fewer targets than did the controls in both the within-modal condition and the cross-modal condition. Target responses to unstudied words were very infrequent in both groups. An ANOVA on the proportion of items recalled revealed a main effect of group $[F(1,30)=4.4, p<.05]$ but no effect of modality $[F(1,30)=2.0, p>.16]$ and no group $\times$ modality interaction $(F<1)$. The amnesic patients were more likely to guess, as evidenced by the fact that they generated more completions (of any kind) to unstudied items $(M=.38)$ than did the controls $(M=.11)$. To take into account this difference, target completions in each condition were expressed as a function of the total number of completions in that condition. The proportion of correctly recalled items as a function of total responses was considerably smaller for the amnesics than for the controls in both the within-modal condition (amnesics, $M=.45$; controls, $M=$ .77 ) and the cross-modal condition (amnesics, $M=.49$; controls, $M=.76)$. An ANOVA on these scores revealed a main effect of group $[F(1,30)=12.6, p<.01]$. No other effects were significant $\left(F_{\mathrm{S}}<1\right)$.

These results replicate and extend previous studies of cross-modal stem completion in amnesia (Carlesimo, Marfia, Loasses, \& Caltagirone, 1996; Graf et al., 1985). Priming was intact in the amnesic patients in the within-

Table 2

Mean Proportion of Stem Completions Unassociated With Memorial Awareness in Experiment 1 for a Subgroup of Amnesic Patients $(n=11)$ Matched to Controls in Terms of Response Bias

\begin{tabular}{lccc}
\hline & \multicolumn{3}{c}{ Study Condition } \\
\cline { 2 - 4 } Group & Visual & Auditory & Unstudied \\
\hline Amnesics & .38 & .16 & .07 \\
Controls & .23 & .11 & .08 \\
\hline
\end{tabular}


Table 3

Mean Proportion of Target Completions Generated in the Cued Recall Task of Experiment 1

\begin{tabular}{lccc}
\hline & \multicolumn{3}{c}{ Study Condition } \\
\cline { 2 - 4 } Group & Visual & Auditory & Unstudied \\
\hline Amnesics & .23 & .20 & .01 \\
Controls & .33 & .28 & .03 \\
\hline
\end{tabular}

modal and in the cross-modal conditions, despite the fact that their cued recall for the same stimuli was markedly deficient. Furthermore, the amnesic patients were impaired not only in their ability to intentionally recall words from the study list but also in their ability to recognize that their implicit stem completions corresponded to words from the study list. Despite this deficit in memorial awareness, their cross-modal priming was intact.

These findings provide strong evidence for the notion that memorial awareness is not necessary for cross-modal priming. Although involuntary conscious memory accompanied cross-modal priming in the control participants (see also Richardson-Klavehn \& Gardiner, 1996), such awareness appears to be merely a correlate, not a prerequisite, for cross-modal priming.

\section{EXPERIMENT 2}

Questions regarding the mechanisms underlying cross-modal priming have recently also been addressed using functional imaging methods. In several PET studies, Badgaiyan, Schacter, and Alpert (1999, 2001; Schacter, Badgaiyan, \& Alpert, 1999) observed that crossmodal, but not within-modal, priming is accompanied by blood flow increases in right anterior prefrontal cortex. Because right prefrontal activation has been observed in a variety of explicit memory tasks (Buckner \& Koutstaal, 1998), these findings raised the possibility that the right frontal activation seen in the cross-modal priming condition might also reflect an aspect of explicit retrieval. To examine this possibility, Badgaiyan et al. (2001) examined cross-modal priming under conditions of divided attention at encoding. They chose to manipulate attention at study because stem completion studies using the process dissociation procedure found that dividing attention reduced the probability of recollection but had no effect on automatic influences of memory (Debner \& Jacoby, 1994; Jacoby et al., 1993). Thus, Badgaiyan et al. (2001) reasoned that if dividing attention abolished the right frontal activation seen in cross-modal priming, this would provide support for the notion that this activation was associated with explicit retrieval. This was indeed the result they obtained. Although prefrontal activation has been implicated in various aspects of explicit retrieval (Rugg \& Wilding, 2000), the authors - in part on the basis of previous behavioral findings (Richardson-Klavehn \& Gardiner, 1996) favored the notion that prefrontal activation reflected involuntary explicit memory (i.e., the memorial awareness that accompanies involuntary retrieval).
Although this interpretation dovetails nicely with the findings of Experiment 1, in which the amnesic patients showed intact cross-modal priming, an aspect of the Badgayian et al. (2001) study appears inconsistent with this interpretation. Namely, in a pilot study, the divided attention condition elicited less priming than did the full attention condition. If, as the authors suggest, the explicit retrieval associated with cross-modal priming (and reflected in frontal activation) is involuntary, it is unclear why priming should be lower under conditions of divided attention than under conditions of full attention. The fact that cross-modal priming was affected by an attentional manipulation suggests to us that cross-modal priming in the full attention condition may have been mediated, at least in part, by voluntary explicit retrieval.

One reason as to why cross-modal priming in the imaging studies, but not the behavioral studies, might be associated with voluntary retrieval lies in methodological differences between these studies. Most notably, in behavioral stem completion studies, studied and unstudied stems are intermixed in the test phase. In contrast, in PET studies, studied and unstudied words must be presented in blocked fashion because of the relatively low temporal resolution of PET. It is possible that presenting all studied words consecutively elicits explicit retrieval strategies that are not otherwise evoked.

In light of this analysis, Experiment 2 examined crossmodal stem completion priming in patients with amnesia under blocked testing conditions that were modeled after those used in the PET studies. We hypothesized that if voluntary retrieval contributes to cross-modal priming under blocked conditions, then amnesic patients should show impaired cross-modal priming in this experiment.

\section{Method}

Participants. Twelve amnesic patients with an etiology of Korsakoff 's syndrome $(n=3)$, anoxia $(n=6)$, encephalitis $(n=2)$, or bithalamic stroke $(n=1)$ participated in this experiment. Ten of these patients had also participated in Experiment 1. The amnesic group had a mean age of 53 years and an average education of 14.4 years. They obtained a mean verbal IQ score of 96 on the WAIS-III. Their memory functioning, as indicated by performance on the WMS-III, was severely compromised: They obtained a general memory index of 58, a visual delay index of 62, and an auditory delay index of 63 . Their attentional abilities, as measured by the WMS-III working memory index, were average $(M=91)$.

The control group consisted of 3 individuals with a history of alcoholism, and 9 individuals without a history of alcoholism. One individual had also participated in Experiment 1. Two participants were replaced because, at the end of the experiment, they indicated that they did not provide the first word that came to mind if they realized that word had appeared on the study list. The control group was matched as closely as possible to the amnesic group in terms of age ( $M=51$ years), education ( $M=15$ years), and verbal IQ $(M=105)$.

Materials and Design. Stimuli consisted of 120 target words and 60 fillers with a mean length of 6.0 letters and a mean word frequency of 96 (Francis \& Kučera, 1982). The three-letter word stem for each word was unique and had at least 10 completions $(M=22.1)$. The 120 target words were divided into two lists, matched for word length, word frequency, and number of possible stem completions. One list was used for the within-modal condition 
and the other for the cross-modal condition. Each list was further divided into two matched sets of 30 items that functioned as studied and unstudied items, respectively. The assignment of lists to conditions was counterbalanced across participants.

Each study list consisted of 30 target items and 15 fillers. Two filler items were presented at the beginning and end of each study list to prevent primacy and recency effects. The other fillers were randomly intermixed with the target items. In accordance with the PET studies, the test lists consisted of two blocks: one block containing stems that corresponded for the most part to studied items and the other block containing stems that corresponded for the most part to unstudied items. The first 9 stems of each block were derived from a mix of studied and unstudied words, and the remaining 21 stems were all derived from studied (or unstudied) words. The order of the studied and unstudied conditions within a test list was counterbalanced across participants.

Procedure. The participants took part in a within-modal task and a cross-modal task that were separated by a 10-min break. Task order was counterbalanced across participants. During the study phase of the within-modal task, stimuli were presented for $2 \mathrm{sec}$ in the center of the computer screen. During the study phase of the cross-modal task, the participants listened to recordings that had been normalized and smoothed using SoundEdit 16. In both tasks, they were asked to rate the pleasantness of each word on a 5-point scale. There was a 3-min break between the study phase and the test phase. The test phase was identical for both tasks. The participants were told that three-letter stems would appear on the screen, and they were asked to complete each stem with the first word that came to mind.

\section{Results and Discussion}

Table 4 presents the proportion of stems completed with targets. The results of the alcoholic and nonalcoholic subgroups were combined because there were no significant differences as a function of alcohol history.

Priming was significant in both groups for both visual modality and auditory modality (all $t \mathrm{~s}>2.1, p \mathrm{~s}<.05$ ). As expected, priming was greater in the within-modal condition than in the cross-modal condition for both groups. Furthermore, whereas within-modal priming was of similar magnitude in the patients and the controls, cross-modal priming was markedly smaller in the patients than in the controls. An ANOVA on priming scores revealed a significant main effect of modality $[F(1,22)=$ $20.0, p<.01]$ and a marginally significant group $\times$ modality interaction $[F(1,22)=3.68, p<.07]$. Crossmodal priming was significantly smaller in the amnesic group than in the control group $[t(12)=2.6, p<.05]$.

Under the blocked presentation conditions of the present experiment, the amnesic patients showed impaired cross-modal priming. This finding is consistent with our hypothesis that voluntary explicit retrieval contributes to cross-modal priming in normal individuals in a blocked paradigm. Our results further suggest that voluntary re-

Table 4

Mean Proportion of Stems Completed With Targets in Experiment 2

\begin{tabular}{lccccc}
\hline & \multicolumn{2}{c}{ Visual } & & \multicolumn{2}{c}{ Auditory } \\
\cline { 2 - 3 } \cline { 5 - 6 } Group & Studied & Unstudied & & Studied & Unstudied \\
\hline Amnesics & .36 & .10 & & .19 & .12 \\
Controls & .39 & .13 & & .26 & .08 \\
\hline
\end{tabular}

trieval does not play a role in within-modal priming, since the amnesics' priming in the visual condition was fully intact.

\section{GENERAL DISCUSSION}

Our findings illustrate that the test format in which stems are presented affects the processes mediating cross-modal priming. In Experiment 1, in which stems corresponding to studied and unstudied words were intermixed, the amnesic patients showed intact within- and cross-modal priming, even though they did not recognize their studied completions. Under these conditions, voluntary retrieval did not play a role in cross-modal priming. Moreover, it was shown that memorial awareness (often associated with involuntary retrieval in normal individuals) is not necessary for intact cross-modal priming. In Experiment 2, in which stems corresponding to studied and unstudied words were blocked, the amnesic patients showed intact within-modal priming, but impaired crossmodal priming, suggesting that a blocked format induced voluntary retrieval strategies in the normal participants. ${ }^{2}$ By inference, we conclude that the right prefrontal activation observed in imaging studies of cross-modal priming likely reflects voluntary, rather than involuntary, explicit memory processes.

The fact that voluntary retrieval mechanisms were recruited in the cross-modal condition of Experiment 2, but not in the within-modal condition, is surprising given that explicit memory is equally available in the two conditions, as demonstrated by the cued recall results of Experiment 1 . Why then is explicit memory more likely to be used in cross-modal stem completion than in withinmodal stem completion? One possibility is that the availability of visual perceptual fluency in the within-modal condition obviates the need for more strategic approaches to the task - the generation of completions for the stems may be driven effortlessly by the perceptual fluency of the target response. Because such fluency is not available in the cross-modal condition, participants may resort to a variety of other strategies to complete the stems, including (but not limited to) a consideration of words that had appeared in the prior study list.

In theory, such voluntary retrieval could arise as a strategy for cross-modal completion regardless of whether stems are presented in blocked or mixed format. In the blocked condition, however, the strategy is reinforced by the fact that words from the study list form appropriate completions for most of the stems within a studied test block. In the mixed condition, by contrast, the strategy is likely to be rejected because words from the study list do not provide appropriate completions for many of the stems in the test list. To examine whether the use of a voluntary retrieval strategy was affected by the likelihood of its initial success, we compared priming in Experiment 2 for those control participants who completed studied stems first and those who completed baseline stems first. Cross-modal priming was greater when studied stems, 
rather than baseline stems, were completed first (.27 vs. .10) $[t(6)=4.1, p<.01]$. (Within-modal priming did not differ as a function of test order; $M=.27$ vs. .26. $)^{3}$ These findings are consistent with the notion that an explicit memory strategy is adopted only when the success of the strategy is clear to the participant, as it is in the blocked condition.

In summary, the present findings suggest that voluntary retrieval mechanisms contribute to stem completion priming when two conditions are met: (1) an alternative heuristic (e.g., perceptual fluency) that provides a less effortful way to perform the task is unavailable, and (2) the success of the strategy is apparent at an early point in the task.

Our findings and interpretation are consistent with the proposal by Schacter and Badgayian (2001) that there are two routes to cross-modal priming - one implicit and one explicit. Priming by means of an explicit route may be elicited by certain task conditions, as outlined above (for other conditions, see Hamnann \& Squire, 1996, and Squire, Shimamura, \& Graf, 1987). However, whereas Schacter and Badgayian (2001) have suggested that these routes may be interchangeable, such that one may substitute for the other, the present findings suggest that, in some conditions, they jointly influence performance. It is under those conditions that performance is impaired in amnesia.

Finally, our analysis has focused specifically on crossmodal priming in the stem completion task. It remains to be seen whether cross-modal priming in other tasks, such as fragment completion, can be accounted for in a similar way. Blum and Yonelinas (2001) recently provided evidence suggesting that cross-modal priming in fragment completion may be mediated differently from cross-modal priming in stem completion, since only participants who claimed to have used explicit retrieval processes showed cross-modal priming in the fragment completion task. At odds with their data, however, is the finding of intact cross-modal fragment completion priming in a group of amnesic patients (Vaidya, Gabrieli, Keane, \& Monti, 1995; but see Kohler et al., 1997, for evidence of impairment in a single case). Clearly, future studies will be needed to analyze the nature of cross-modal priming in fragment completion and to compare cross-modal priming in the fragment completion task and the stem completion task.

\section{REFERENCES}

Badgaiyan, R. D., Schacter, D. L., \& Alpert, N. M. (I999). Auditory priming within and across modalities: Evidence from positron emission tomography. Journal of Cognitive Neuroscience, 11, 337 348.

Badgaiyan, R. D., Schacter, D. L., \& Alpert, N. M. (2001). Priming within and across modalities: Exploring the nature of rCBF increases and decreases. NeuroImage, 13, 272-282.

Bassili, J. N., Smith, M. C., \& Macleod, M. C. (1989). Auditory and visual word-stem completion: Separating data-driven and conceptually driven processes. Quarterly Journal of Experimental Psychology, 41A, 439-452.

Blum, D., \& Yonelinas A. P. (2001). Transfer across modality in perceptual implicit memory. Psychonomic Bulletin \& Review, 8, 147-154.

BucKner, R. L., \& KoutstaAl, W. (1998). Functional neuroimaging studies of encoding, priming and explicit memory retrieval. Proceedings of the National Academy of Sciences, 95, 891-898.

CArlesimo, G. A. (1994). Perceptual and conceptual priming in amnesic and alcoholic patients. Neuropsychologia, 32, 903-922.

Carlesimo, G. A., Marfia, G. A., Loasses, A., \& Caltagirone, C. (1996). Perceptual and conceptual components in implicit and explicit stem completion. Neuropsychologia, 34, 785-792.

Craik, F., Moscovitch, M., \& McDowd, J. (1994). Contributions of surface and conceptual information to performance on implicit and explicit memory tasks. Journal of Experimental Psychology: Learning, Memory, \& Cognition, 20, 864-875.

Curran, T., Schacter, D. L., \& Galluccio, L. (1999). Cross-modal priming and explicit memory in patients with verbal production deficits. Brain \& Cognition, 39, 133-146.

Debner, J. A., \& JACoBy, L. L. (1994). Unconscious perception: Attention, awareness, and control. Journal of Experimental Psychology: Learning, Memory, \& Cognition, 20, 304-317.

Francis, W. N., \& KuČERA, H. (1982). Frequency analysis of English usage: Lexicon and grammar. Boston: Houghton Mifflin.

Graf, P., Shimamura, A. P., \& SQuire, L. R. (1985). Priming across modalities and across category levels: Extending the domain of preserved function in amnesia. Journal of Experimental Psychology: Learning, Memory, \& Cognition, 11, 385-395.

Hamnann, S. B., \& SQuire, L. R. (1996). Level-of-processing effects in word-completion priming: A neuropsychological study. Journal of Experimental Psychology: Learning, Memory, \& Cognition, 22, 933 947.

JAcoby, L. L., Toth, J. P., \& Yonelinas, A. P. (1993). Separating conscious and unconscious influences of memory: Measuring recollection. Journal of Experimental Psychology: General, 122, 139-154.

Jacoby, L. L., Toth, J. P., Yonelinas, A. P., \& Debner, J. A. (1994). The relationship between conscious and unconscious influences: Independence or redundancy? Journal of Experimental Psychology: General, 123, 216-219.

Kirsner, K., Dunn, J. C., \& Standen, P. (1989). Domain-specific resources in word recognition. In S. Lewandowsky, J. C. Dunn, \& K. Kirsner (Eds.), Implicit memory: Theoretical issues (pp. 99-122). Hillsdale, NJ: Erlbaum.

Kohler, S., Habib, R, Black, S. E., Szekely, C., Sinden, M., \& TulVING, E. (1997). Cross modality priming in a densely amnesic subject K.C. Brain \& Cognition, 35, 420-423.

Moscovitch, M., Vriezen, E., \& GotTstein, J. (1993). Implicit tests of memory in patients with focal lesions or degenerative brain disorders. In H. Spinnler \& F. Boller (Eds.), Handbook of neuropsychology (Vol. 8, pp. 133-173). Amsterdam: Elsevier.

Richardson-Klavehn, A., \& Gardiner, J. M. (1996). Cross-modality priming in stem completion reflects conscious memory, but not voluntary memory. Psychonomic Bulletin \& Review, 3, 238-244.

RichaRdSON-KLAVEHN, A., GARDINER, J. M., \& JAVA, R. I. (1994). Involuntary conscious memory and the method of opposition. Memory, 2 , $1-29$.

Roediger, H. L., III, Weldon, M. S., \& Challis, B. H. (1989). Explaining dissociations between implicit and explicit measures of retention: A processing account. In H. L. Roediger III \& F. I. M. Craik (Eds.), Varieties of memory and consciousness: Essays in honour of Endel Tulving (pp. 3-41). Hillsdale, NJ: Erlbaum.

RugG, M. D., \& Wilding, E. L. (2000). Retrieval processing and episodic memory. Trends in Cognitive Sciences, 4, 108-115.

Schacter, D. L. (1994). Priming and multiple memory systems: Perceptual mechanisms of implicit memory. In D. L. Schacter \& E. Tulving (Eds.), Memory Systems 1994 (pp. 233-268). Cambridge, MA: MIT Press.

SCHACTER,D. L., \& BADGAIYAN, R. D. (2001). Neuroimaging of priming: New perspectives on implicit and explicit memory. Current Directions in Psychological Science, 10, 1-4.

Schacter, D. L., Badgaiyan, R. D., \& Alpert, N. M. (1999). Visual word stem completion priming within and across modalities: A PET study. NeuroReport, 10, 2061-2065.

SCHACTER, D. L., Bowers, J., \& BoOKER, J. (1989). Intention, awareness, and implicit memory: The retrieval intentionality criterion. In S. Lew- 
andowsky, J. C. Dunn, \& K. Kirsner (Eds.), Implicit memory: Theoretical issues (pp. 47-65). Hillsdale, NJ: Erlbaum.

Schacter, D. L., Chiu, P. C. Y., \& OChSNer, K. N. (1993). Implicit memory: A selective review. Annual Review of Neuroscience, 16, 159-182.

Squire, L., Shimamura, A., \& Graf, P. (1987). Strength and duration of priming effects in normal subjects and amnesic patients. Neuropsychologia, 25, 195-210.

Toth, J. P., \& Reingold, E. M. (1996). Beyond perception: Conceptual contributions to unconscious influences of memory. In G. Underwood (Ed.), Implicit cognition (pp. 41-84). Oxford: Oxford University Press.

Tulving, E., \& Schacter, D. L. (1990). Priming and human memory systems. Science, 247, 301-306.

Vaidya, C. J., Gabrieli, J. D. E., Keane, M. M., \& Monti, L. A. (1995). Perceptual and conceptual memory processes in global amnesia. Neuropsychology, 9, 580-591.

WECHSLER, D. (1997a). Wechsler Adult Intelligence Scale-III. San Antonio, TX: Psychological Corporation.

WeCHSLER, D. (1997b) Wechsler Memory Scale-III. San Antonio, TX: Psychological Corporation.

\section{NOTES}

1. Cross-modal priming unassociated with awareness was smaller in the group of amnesics as a whole (because of the overestimation of priming with awareness) but was nonetheless significant.

2. A reviewer questioned whether the contribution of explicit memory to cross-modal priming in Experiment 2, but not in Experiment 1, might have been due to the fact that the study-test delay was shorter in Experiment 2 (3 vs. $6 \mathrm{~min}$.), rather than to the blocking manipulation. We do not believe this to be the case, since explicit memory did not make a contribution to cross-modal priming in the study of Carlesimo (1994), in which the study-test delay was only $1-2 \mathrm{~min}$, but stems were intermixed at test.

3. As expected, test order did not affect the magnitude of priming in the amnesic patients in the cross-modal condition or in the withinmodal condition.

(Manuscript received March 19, 2001; revision accepted for publication July 16, 2001.) 